\title{
Sweat Gland Carcinoma
}

National Cancer Institute

\section{Source}

National Cancer Institute. Sweat Gland Carcinoma. NCI Thesaurus. Code C6938.

A carcinoma arising from the sweat glands. Representative examples include tubular carcinoma, spiradenocarcinoma, eccrine carcinoma, hidradenocarcinoma, and apocrine carcinoma. 